\title{
Algorithm refinement for fluctuating hydrodynamics
}

\author{
Sarah A. Williams \\ Department of Mathematics \\ University of California, Davis \\ Davis, California, 95616, USA
}

John B. Bell

Center for Computational Sciences and Engineering

Lawrence Berkeley National Laboratory

Berkeley, California, 94720, USA

\author{
Alejandro L. Garcia \\ Department of Physics \\ San Jose State University \\ San Jose, California, 95192, USA
}

July 6, 2007

\begin{abstract}
This paper introduces an adaptive mesh and algorithm refinement method for fluctuating hydrodynamics. This particle-continuum hybrid simulates the dynamics of a compressible fluid with thermal fluctuations. The particle al-
\end{abstract}


gorithm is direct simulation Monte Carlo (DSMC), a molecular-level scheme based on the Boltzmann equation. The continuum algorithm is based on the Landau-Lifshitz Navier-Stokes (LLNS) equations, which incorporate thermal fluctuations into macroscopic hydrodynamics by using stochastic fluxes. It uses a recently-developed solver for LLNS, based on third-order Runge-Kutta. We present numerical tests of systems in and out of equilibrium, including timedependent systems, and demonstrate dynamic adaptive refinement by the computation of a moving shock wave. Mean system behavior and second moment statistics of our simulations match theoretical values and benchmarks well. We find that particular attention should be paid to the spectrum of the flux at the interface between the particle and continuum methods, specifically for the non-hydrodynamic (kinetic) time scales.

\section{Introduction}

Adaptive mesh refinement (AMR) is often employed in computational fluid dynamics (CFD) simulations to improve efficiency and/or accuracy: a fine mesh is applied in regions where high resolution is required for accuracy, and a coarser mesh is applied elsewhere to moderate computational cost. For dynamic problems, the area that is a candidate for mesh refinement may change over time, so methods have been developed to adaptively identify the refinement target area at each time step (e.g., [1, 2, 3]).

However, at the smallest scales, on the order of a molecular mean free path, continuum assumptions may not hold, so CFD approaches do not accurately model the relevant physics. In such a regime, adaptive mesh and algorithm refinement (AMAR) improves on AMR by introducing a more physicallyaccurate particle method to replace the continuum solver on the finest mesh. An improved simulation does not result from continued refinement of the mesh, but rather "refinement" of the algorithm, i.e., switching from the continuum model to a particle simulation. Introduced in [4], AMAR has proved to be a useful paradigm for multiscale fluid modeling. In this paper, we describe AMAR for fluctuating hydrodynamics. 
Random thermal fluctuations occur in fluids at microscopic scales (consider Brownian motion), and these microscopic fluctuations can lead to macroscopic system effects. The correct treatment of fluctuations is especially important for stochastic, nonlinear systems, such as those undergoing phase transitions, nucleation, noise-driven instabilities, and combustive ignition. In these and related applications, nonlinearities can exponentially amplify the influence of the fluctuations. As an example, consider the classical Rayleigh-Taylor problem and the related Richtmyer-Meshkov instability that are prototypical problems for the study of turbulent mixing. A heavy fluid sits above a light fluid, and spontaneous microscopic fluctuation at the interface between the fluids leads to turbulent mixing throughout the domain. Kadau and co-workers have recently studied the development of this turbulence at the atomic scale $[5,6]$. That group's atomistic simulations indicate that thermal fluctuations are an important driver of the behavior of complex flows, certainly at the smallest scales and perhaps at all scales. For example, in stochastic atomistic simulations of Rayleigh-Taylor, and in laboratory experiments, spikes of the heavy fluid that project into the light fluid can break off to form isolated droplets; this phenomenon cannot be reproduced accurately by deterministic continuum models. However, the physical and temporal domain on which this type of atomistic simulation is computationally feasible is extremely limited (less than a billion atoms per nanosecond) given current algorithms and near-term computational power. The goal of AMAR for fluctuating hydrodynamics is to effectively enhance the computing power available for investigations of this type of phenomenon.

Hadjiconstantinou has reviewed theoretical and numerical approaches to challenges arising from the breakdown of the Navier-Stokes description at small-scale and (with Wijesinghe) described a variety of particle-continuum methods for multiscale hydrodynamics [7, 8]. The work presented here is the latest effort in a line of work that has focussed on building AMAR hybrids for flows of increasing sophistication. A hybrid coupling Navier-Stokes and DSMC was developed in [4], with several of the technical issues necessary for implementation extended in [9]. Stochastic hybrid methods were developed in [10] (mass diffusion), [11] (the "train model" for momentum diffusion), and 
[12] (Burgers' equation). Other recent work on coupling particle and continuum methods includes [13] (DSMC and Navier-Stokes, for aerospace applications), [14] (molecular dynamics and isothermal fluctuating hydrodynamics, for polymer simulations), and [15] (an adaptive refinement approach based on a direct numerical solution of the Boltzmann transport equation and kinetic continuum schemes).

The AMAR approach is characterized by several design principles. In contrast to other algorithm refinement (AR) approaches (see, e.g. [16]), in AMAR (as in AMR) the solution of the macroscopic model is maintained over the entire domain. A refinement criterion is used to estimate where the improved representation of the particle method is required. That region, which can change dynamically, is then "covered" with a particle patch. In this hierarchical representation, upon synchronization the particle solution replaces the continuum solution in the regions covered by the molecular patches.

Given their complexity, the implementations of hybrid codes benefit greatly from modularization (e.g., see [13]). Another fundamental tenant of the AMAR approach to particle-continuum hybridization is that the coupling of the two algorithms is completely encapsulated in several "hand-shaking" routines. Taken as a unit, the particle method plus these modular routines perform exactly the same function as any fine grid in a single-algorithm AMR method. The encapsulated coupling routines perform the following functions: continuum data is used to generate particles that flow into the particle region; flux across the boundaries of the particle region is recorded and used to correct neighboring continuum values; cell-averaged data from the particle grid replaces data on the underlying continuum grid; continuum data is used to generate particles to initialize new particle regions identified by the refinement criterion

Implementation details are given in the next two sections of the paper. Our continuum approach for fluctuating hydrodynamics is an explicit finite volume method for solving the Landau-Lifshitz Navier-Stokes equations for compressible fluid flow (see Section 2.1), and, as noted above, the particle method is direct simulation Monte Carlo (DSMC) (see Section 2.2). Hybrid coupling details are discussed in Section 3. Numerical results for problems with a 
static refinement region are presented in Section 4, for a variety of steady-state and time-dependent problems with the flow restricted to one spatial dimension. (Forthcoming work will illustrate this construction extended to 2- and 3-dimensional systems.) Details of adaptive refinement are discussed in Section 4.5, including numerical results for an adaptive refinement shock-tracking problem. We conclude, in Section 5, with a discussion of future work.

\section{Components of the Hybrid}

\subsection{Continuum Approach}

The continuum model and solver discussed in this section was introduced in [17], and the reader is referred to that paper for further details of the method and measurements of its performance.

To incorporate thermal fluctuations into macroscopic hydrodynamics, Landau and Lifshitz introduced an extended form of the Navier-Stokes equations by adding stochastic flux terms [18]. The Landau-Lifshitz Navier-Stokes (LLNS) equations may be written as

$$
\partial \mathbf{U} / \partial t+\nabla \cdot \mathbf{F}=\nabla \cdot \mathbf{D}+\nabla \cdot \mathbf{S}
$$

where

$$
\mathbf{U}=\left(\begin{array}{c}
\rho \\
\mathbf{J} \\
E
\end{array}\right)
$$

is the vector of conserved quantities (density of mass, momentum and energy).

The hyperbolic flux is given by

$$
\mathbf{F}=\left(\begin{array}{c}
\rho \mathbf{v} \\
\rho \mathbf{v} \mathbf{v}+P \mathbf{I} \\
(E+P) \mathbf{v}
\end{array}\right)
$$

and the diffusive flux is given by

$$
\mathbf{D}=\left(\begin{array}{c}
0 \\
\tau \\
\tau \cdot \mathbf{v}+\kappa \nabla T
\end{array}\right),
$$


where $\mathbf{v}$ is the fluid velocity, $P$ is the pressure, $T$ is the temperature, and $\tau=\eta\left(\nabla \mathbf{v}+\nabla \mathbf{v}^{T}-\frac{2}{3} \mathbf{I} \nabla \cdot \mathbf{v}\right)$ is the stress tensor. Here $\eta$ and $\kappa$ are coefficients of viscosity and thermal conductivity, respectively, where we have assumed the bulk viscosity is zero.

The mass flux is microscopically exact but the other two flux components are not; for example, at molecular scales heat may spontaneously flow from cold to hot, in violation of the macroscopic Fourier law. To account for such spontaneous fluctuations, the LLNS equations include a stochastic flux

$$
\mathbf{S}=\left(\begin{array}{c}
0 \\
\mathcal{S} \\
\mathcal{Q}+\mathbf{v} \cdot \mathcal{S}
\end{array}\right)
$$

where the stochastic stress tensor $\mathcal{S}$ and heat flux $\mathcal{Q}$ have zero mean and covariances given by

$$
\begin{gathered}
\left\langle\mathcal{S}_{i j}(\mathbf{r}, t) \mathcal{S}_{k \ell}\left(\mathbf{r}^{\prime}, t^{\prime}\right)\right\rangle=2 k_{B} \eta T\left(\delta_{i k}^{K} \delta_{j \ell}^{K}+\delta_{i \ell}^{K} \delta_{j k}^{K}-\frac{2}{3} \delta_{i j}^{K} \delta_{k \ell}^{K}\right) \delta\left(\mathbf{r}-\mathbf{r}^{\prime}\right) \delta\left(t-t^{\prime}\right) \\
\left\langle\mathcal{Q}_{i}(\mathbf{r}, t) \mathcal{Q}_{j}\left(\mathbf{r}^{\prime}, t^{\prime}\right)\right\rangle=2 k_{B} \kappa T^{2} \delta_{i j}^{K} \delta\left(\mathbf{r}-\mathbf{r}^{\prime}\right) \delta\left(t-t^{\prime}\right)
\end{gathered}
$$

and

$$
\left\langle\mathcal{S}_{i j}(\mathbf{r}, t) \mathcal{Q}_{k}\left(\mathbf{r}^{\prime}, t^{\prime}\right)\right\rangle=0
$$

where $k_{B}$ is Boltzmann's constant. The LLNS equations have been derived by a variety of approaches (see $[18,19,20,21]$ ) and have even been extended to relativistic hydrodynamics [22]. While they were originally developed for equilibrium fluctuations, specifically the Rayleigh and Brillouin spectral lines in light scattering, the validity of the LLNS equations for non-equilibrium systems has been derived [23] and verified in molecular simulations [24, 25, 26].

For simplification, in this work we restrict our attention to flow in one dimension. That is, we take the fluid velocity $\mathbf{v}=(u, v, w)$ to be threedimensional, but we only consider spatial derivatives in the x-direction. Then 
(1) simplifies to

$$
\begin{aligned}
& \frac{\partial}{\partial t}\left(\begin{array}{c}
\rho \\
\rho u \\
\rho v \\
\rho w \\
E
\end{array}\right)=-\frac{\partial}{\partial x}\left(\begin{array}{c}
\rho u \\
\rho u^{2}+P \\
\rho u v \\
\rho u w \\
(E+P) u
\end{array}\right)+\frac{\partial}{\partial x}\left(\begin{array}{c}
0 \\
\tau_{11} \\
\tau_{12} \\
\tau_{13} \\
\tau_{11} u+\tau_{12} v+\tau_{13} w+\kappa \partial_{x} T
\end{array}\right) \\
& +\frac{\partial}{\partial x}\left(\begin{array}{c}
0 \\
s_{11} \\
s_{12} \\
s_{13} \\
q+u s_{11}+v s_{12}+w s_{13}
\end{array}\right)
\end{aligned}
$$

where $s_{11}, s_{12}, s_{13}$, and $q$ are independent random variables with zero mean and variances,

$$
\begin{aligned}
& \left\langle s_{11}(x, t) s_{11}\left(x^{\prime}, t^{\prime}\right)\right\rangle=\frac{8 k_{B} \eta T}{3 \sigma} \delta\left(x-x^{\prime}\right) \delta\left(t-t^{\prime}\right), \\
& \left\langle s_{12}(x, t) s_{12}\left(x^{\prime}, t^{\prime}\right)\right\rangle=\frac{2 k_{B} \eta T}{\sigma} \delta\left(x-x^{\prime}\right) \delta\left(t-t^{\prime}\right), \\
& \left\langle s_{13}(x, t) s_{13}\left(x^{\prime}, t^{\prime}\right)\right\rangle=\frac{2 k_{B} \eta T}{\sigma} \delta\left(x-x^{\prime}\right) \delta\left(t-t^{\prime}\right),
\end{aligned}
$$

and

$$
\left\langle q(x, t) q\left(x^{\prime}, t^{\prime}\right)\right\rangle=\frac{2 k_{B} \kappa T^{2}}{\sigma} \delta\left(x-x^{\prime}\right) \delta\left(t-t^{\prime}\right),
$$

with $\sigma$ being the surface area of the system in the $y z$-plane.

For the calculations described in this paper take the fluid to be a dilute gas with equation of state $P=\rho R T$ (ideal gas law) and energy density $E=c_{v} \rho T+\frac{1}{2} \rho\left(u^{2}+v^{2}+w^{2}\right)$. The transport coefficients are only functions of temperature, specifically we take them as $\eta=\eta_{0} \sqrt{T}$ and $\kappa=\kappa_{0} \sqrt{T}$, where the constants $\eta_{0}$ and $\kappa_{0}$ are chosen to match the viscosity and thermal conductivity of a hard sphere gas. We also have gas constant $R=k_{B} / m$ and $c_{v}=\frac{R}{\gamma-1}$ where $m$ is the mass of a particle and the ratio of specific heats is taken to be $\gamma=\frac{5}{3}$, that is, a monatomic gas. Note that generalizations of fluid parameters are straight-forward and the choice of a monatomic hard sphere gas is for convenience in matching parameters in the PDE with those of DSMC simulations (see section 2.2). 
The principal difficulty in solving Eqs.(2) arises because there is no stochastic forcing term in the mass conservation equation. Accurately capturing density fluctuations requires that the fluctuations be preserved in computing the mass flux. Another key observation is that the representation of fluctuations in computational fluid dynamics schemes is also sensitive to the time step, with extremely small time steps leading to improved results. This suggests that temporal accuracy also plays a significant role in capturing fluctuations. Based on these observations, a discretization aimed specifically at capturing fluctuations in the LLNS equations has been developed [17]. The method is based on a third-order, total variation diminishing (TVD) Runge-Kutta temporal integrator (RK3) [27, 28] combined with a centered discretization of hyperbolic and diffusive fluxes.

The RK3 discretizaton can be written in the following three-stage form:

$$
\begin{aligned}
\mathbf{U}_{j}^{n+1 / 3} & =\mathbf{U}_{j}^{n}-\frac{\Delta t}{\Delta x}\left(\mathcal{F}_{j+1 / 2}^{n}-\mathcal{F}_{j-1 / 2}^{n}\right) \\
\mathbf{U}_{j}^{n+2 / 3} & =\frac{3}{4} \mathbf{U}_{j}^{n}+\frac{1}{4} \mathbf{U}_{j}^{n+1 / 3}-\frac{1}{4}\left(\frac{\Delta t}{\Delta x}\right)\left(\mathcal{F}_{j+1 / 2}^{n+1 / 3}-\mathcal{F}_{j-1 / 2}^{n+1 / 3}\right) \\
\mathbf{U}_{j}^{n+1} & =\frac{1}{3} \mathbf{U}_{j}^{n}+\frac{2}{3} \mathbf{U}_{j}^{n+2 / 3}-\frac{2}{3}\left(\frac{\Delta t}{\Delta x}\right)\left(\mathcal{F}_{j+1 / 2}^{n+2 / 3}-\mathcal{F}_{j-1 / 2}^{n+2 / 3}\right),
\end{aligned}
$$

where $\mathcal{F}^{m}=-\mathbf{F}\left(\mathbf{U}^{m}\right)+\mathbf{D}\left(\mathbf{U}^{m}\right)+\tilde{\mathbf{S}}\left(\mathbf{U}^{m}\right)$ and $\tilde{\mathbf{S}}=\sqrt{2} \mathbf{S}$. The diffusive terms $\mathbf{D}$ are discretized with standard second-order finite difference approximations. For $\tilde{\mathbf{S}}_{j+1 / 2}$, the approximation to the stochastic stress tensor, is computed as

$$
s_{m n}=\sqrt{\frac{k_{B}}{\Delta t V_{c}}\left(1+\frac{1}{3} \delta_{m n}^{K}\right)\left(\eta_{j+1} T_{j+1}+\eta_{j} T_{j}\right)} \Re_{j+1 / 2}
$$

where $V_{c}=\sigma \Delta x$ is the volume of a cell and the $\Re$ 's are independent, Gaussian distributed random values with zero mean and unit variance. Similarly, the discretized stochastic heat flux is evaluated as

$$
q=\sqrt{\frac{k_{B}}{\Delta t V_{c}}\left(\kappa_{j+1}\left(T_{j+1}\right)^{2}+\kappa_{j}\left(T_{j}\right)^{2}\right)} \Re_{j+1 / 2} .
$$

Note that $\tilde{\mathbf{S}}$ is evaluated using the instantaneous values of the state variables, i.e., the noise here is multiplicative. In [17] the effect of this multiplicity was found to be negligible.

Combining the three stages, we can write

$$
\mathbf{U}_{j}^{n+1}=\mathbf{U}_{j}^{n}-\frac{\Delta t}{\Delta x}\left(\mathcal{F}_{j+1 / 2}^{\Sigma}-\mathcal{F}_{j-1 / 2}^{\Sigma}\right) .
$$


where

$$
\mathcal{F}_{j \pm 1 / 2}^{\Sigma}=\frac{1}{6} \mathcal{F}_{j \pm 1 / 2}^{n}+\frac{1}{6} \mathcal{F}_{j \pm 1 / 2}^{n+1 / 3}+\frac{2}{3} \mathcal{F}_{j \pm 1 / 2}^{n+2 / 3}
$$

The variance in the stochastic flux at $j+1 / 2$ is given by

$$
\begin{aligned}
\left\langle\left(\mathbf{S}_{j+1 / 2}^{\Sigma}\right)^{2}\right\rangle & =\left\langle\left(\frac{1}{6}\left(\tilde{\mathbf{S}}_{j+1 / 2}^{n}\right)+\frac{1}{6}\left(\tilde{\mathbf{S}}_{j+1 / 2}^{n+1 / 3}\right)+\frac{2}{3}\left(\tilde{\mathbf{S}}_{j+1 / 2}^{n+2 / 3}\right)\right)^{2}\right\rangle \\
& =\left(\frac{1}{6}\right)^{2}\left\langle\left(\tilde{\mathbf{S}}_{j+1 / 2}^{n}\right)^{2}\right\rangle+\left(\frac{1}{6}\right)^{2}\left\langle\left(\tilde{\mathbf{S}}_{j+1 / 2}^{n+1 / 3}\right)^{2}\right\rangle+\left(\frac{2}{3}\right)^{2}\left\langle\left(\tilde{\mathbf{S}}_{j+1 / 2}^{n+2 / 3}\right)^{2}\right\rangle
\end{aligned}
$$

Neglecting the multiplicity in the noise we obtain the desired result that $\left\langle\left(\mathbf{S}^{\Sigma}\right)^{2}\right\rangle=$ $\frac{1}{2}\left\langle(\tilde{\mathbf{S}})^{2}\right\rangle=\left\langle(\mathbf{S})^{2}\right\rangle$, that is, taking $\tilde{\mathbf{S}}=\sqrt{2} \mathbf{S}$ corrects for the reduction of the stochastic flux variance due to the three-stage averaging of the fluxes. However, this treatment does not directly affect the fluctuations in density, since the component of $\mathbf{S}$ in the continuity equation is zero. The density fluctuations are controlled by the spatial discretization. To compensate for the suppression of density fluctuations due to the temporal averaging we interpolate the momentum $J=\rho u$ (and the other conserved quantities) from cell-centered values:

$$
J_{j+1 / 2}=\alpha_{1}\left(J_{j}+J_{j+1}\right)-\alpha_{2}\left(J_{j-1}+J_{j+2}\right),
$$

where

$$
\alpha_{1}=(\sqrt{7}+1) / 4 \quad \text { and } \quad \alpha_{2}=(\sqrt{7}-1) / 4 .
$$

Then in the case in which $J$ is statistically stationary and constant in space we have exactly $J_{j+1 / 2}=J$ and $\left\langle\delta J_{j+1 / 2}^{2}\right\rangle=2\left\langle\delta J^{2}\right\rangle$, as desired; the interpolation is consistent and compensates for the variance-reducing effect of the multi-stage Runge-Kutta algorithm.

\subsection{Particle Approach}

The particle method used here is the direct simulation Monte Carlo (DSMC) algorithm, a well-known method for computing gas dynamics at the molecular scale; see $[29,30]$ for pedagogical expositions on DSMC, [31] for a complete reference, and [32] for a proof of the method's equivalence to the Boltzmann equation. As in molecular dynamics, the state of the system in DSMC is given by the positions and velocities of particles. In each time step, the particles are first moved as if they did not interact with each other. After moving the 
particles and imposing any boundary conditions, collisions are evaluated by a stochastic process, conserving momentum and energy and selecting the postcollision angles from their kinetic theory distributions.

While DSMC is a stochastic algorithm the statistical variation of the physical quantities has nothing to do with the "Monte Carlo" portion of the method. Equilibrium fluctuations are correctly simulated by DSMC in the same fashion as in molecular dynamics simulations, specifically, by the fact that both algorithms produce the correct density of states for the appropriate equilibrium ensembles. For example, for a dilute gas the velocity distribution of the particles is the Maxwell-Boltzmann distribution and the positions are uniformly distributed. For both equilibrium and non-equilibrium problems DSMC yields the physical spectra of spontaneous thermal fluctuations, as confirmed by excellent agreement with fluctuating hydrodynamic theory $[33,25,24]$ and molecular dynamics simulations $[34,26]$.

In this work the simulated physical system is a dilute monatomic hardsphere gas. For engineering applications more realistic molecular models are regularly used in DSMC; for such a case the construction presented here would only be modified by adjusting the functional form of the transport coefficients and including internal degrees of freedom in the total energy. Our simulation geometry is a rectangular volume with periodic boundary conditions in the $y$

and $z$ directions. In the $x$ direction, Dirichlet (or "particle reservoir") boundary conditions are used to couple the DSMC domain to the continuum domain of our hybrid method. These interface conditions are described in the next section.

\section{$3 \quad$ Hybrid implementation}

The fundamental goal of the algorithm refinement hybrid is to represent the fluid dynamics with the low-cost continuum model everywhere except in a localized region where higher-fidelity particle representation is required. In this section, we assume that a fixed refinement region is identified a priori. Additional considerations necessary for dynamic refinement are discussed in Section 4.5.

The coupling between the particle and continuum regions uses the ana- 
$\log$ of constructs used in developing hierarchical mesh refinement algorithms. The continuum method is applied to the entire computational domain, and a particle region, or patch, is overlaid in refinement regions. For simplicity, in this discussion we will assume that there is a single refined patch. Generalization of the approach to include multiple patches (e.g. [9]) is fairly straightforward.

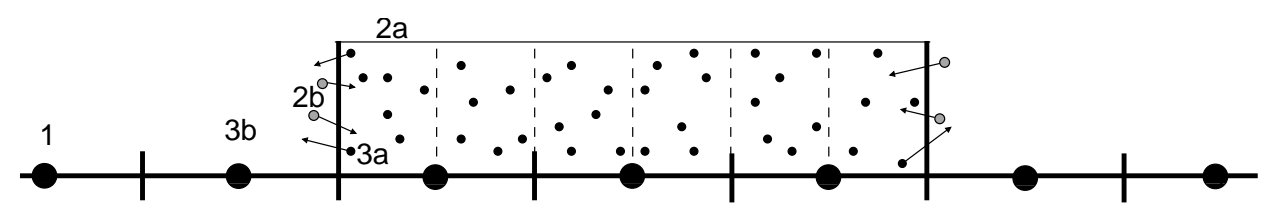

Figure 1: Schematic representation of the coupling mechanisms of the hybrid algorithm. 1. Advance continuum solution. 2. Advance DSMC solution (2a), using continuum data in reservoir boundaries $(2 \mathrm{~b})$. 3. Reflux (3a) to correct continuum solution near interface (3b).

Integration on the hierarchy is a three step process, as depicted in Figure 1. First (1), we integrate the continuum algorithm from $t^{n}$ to $t^{n+1}$, i.e., for a continuum step $\Delta t$. Next (2), the particle simulation is advanced to time $t^{n+1}$. Continuum data at the edge of the particle patch provides reservoir boundary conditions for the particle method. The implementation of reservoir boundary conditions for DSMC is described in [4]. As in that paper, particles that enter the particle patch have velocities drawn from the either the Maxwell-Boltzmann distribution or the Chapman-Enskog distribution. While the Chapman-Enskog distribution is preferred in deterministic hybrids (see [4]) we find that in the stochastic hybrid the Maxwell-Boltzmann distribution sometimes yields better results for the second moment statistics (see Sections 4.1 and 4.3). While Chapman-Enskog yields slightly more accurate results for time-dependent problems, where we focus on the mean behavior of the system (see Sections 4.4 and 4.5), one must recall that the derivation of the LLNS equations is based on the assumption of local equilibrium (e.g., gradients do not appear in the amplitudes of the stochastic fluxes). We return to this point in Section 5.

When particle velocities in the reservoir cells are generated from the Chapman-Enskog distribution, the gradients of fluid velocity and temperature must be estimated in those cells. Furthermore, we also account for density gra- 
dients and generate the particle positions in the reservoir cells accordingly (see the Appendix). However, since the fluctuating continuum model generates steep local gradients, even at equilibrium, we use a regional gradient estimate to represent underlying gradient trends. The regional gradient $D(\xi)$ is implemented as:

$$
D(\xi)_{j}=\frac{1}{S \Delta x}\left[\frac{1}{S} \sum_{i=1}^{S} \xi_{j+i}-\frac{1}{S} \sum_{i=1}^{S} \xi_{j-(i-1)}\right],
$$

where $\xi$ is one of the conserved quantities and $S$ indicates the width of the gradient stencil (we use $S=6$ ). Because the Chapman-Enksog distribution is derived from a perturbation expansion in dimensionless gradient we use slopelimiting to bound the breakdown parameter (see [35] for details).

In general, DSMC uses smaller space and time increments than the continuum method. Spatial refinement is accomplished by dividing the DSMC patch into any number of smaller cells at the collision stage of the algorithm. For simplicity, we assume that an integer number of time steps elapse on the particle patch for every continuum time step. The old and new continuum states, $U_{j}^{n}$ and $U_{j}^{n+1}$, are retained until all the intermediate particle time steps are complete, and the continuum data is interpolated in time to provide appropriate boundary data at each particle method time step. An alternative version of the DSMC algorithm allows the time steps to be event-driven[12], but here we use time increments of fixed size.

Finally, step (3) corrects the macroscopic solution to reflect the effect of the microscopic model as though the integration were tightly coupled. On the region covered by the particle representation we replace the continuum solution by the more accurate particle representation. That is, for each cell covered by the particle patch we set

$$
\begin{aligned}
\rho_{j}^{n+1} & =\frac{N_{j} m}{\sigma \Delta x} \\
\mathbf{J}_{j}^{n+1} & =\frac{\sum_{N_{j}} \mathbf{v} m}{\sigma \Delta x} \\
E_{j}^{n+1} & =\frac{\sum_{N_{j}} \frac{1}{2}\left(u^{2}+v^{2}+w^{2}\right) m}{\sigma \Delta x},
\end{aligned}
$$

where $N_{j}$ is the number of particles in cell $j, m$ is the mass of a particle, and $\sigma \Delta x$ is the volume of a computational cell. In the calculation of each momentum 
component, the product of the particle mass with the velocity is summed over all particles in the cell. In the calculation of energy we sum the squares of the three velocity components over all the particles in the cell.

Moreover, we must correct ("reflux") the continuum solution in the cells immediately adjacent to the particle region, to account for the gas that entered or exited the particle patch during step (2). Specifically, suppose the leftmost cell of the particle patch is cell $j+1$. The value in continuum cell $j$ was already updated with the continuum stochastic RK3 scheme, using the flux $\mathcal{F}=-\mathbf{F}+\mathbf{D}+\tilde{\mathbf{S}}$ computed from the continuum values. However, this value is not consistent with the microscopic flux given by the net number of particles moving across edge $j+1 / 2$. The reflux step corrects the value in cell- $j$ so that it is consistent with the microscopic flux at $j+1 / 2$.

To perform the refluxing correction we monitor the number of particles, $N_{j+1 / 2}$ and $N_{j+1 / 2}^{\leftarrow}$, that move into and out of the particle region, respectively, across the continuum/particle interface at edge $j+1 / 2$. We then correct the continuum solution as

$$
\mathbf{U}_{j}^{\prime n+1}=\mathbf{U}_{j}^{n+1}+\frac{\Delta t}{\Delta x}\left(\mathcal{F}_{j+1 / 2}^{\Sigma}-\mathcal{F}_{j+1 / 2}^{\mathrm{P}}\right)
$$

where the prime indicates the value after the refluxing update. The net particle flux is

$$
\mathcal{F}_{j+1 / 2}^{\mathrm{P}}=\frac{m}{\sigma \Delta t}\left(\begin{array}{c}
N_{j+1 / 2}-N_{j+1 / 2}^{\leftarrow} \\
\sum_{i}^{\overrightarrow{ }} \mathbf{v}_{i}-\sum_{i}^{\leftarrow} \mathbf{v}_{i} \\
\frac{1}{2} \sum_{i}^{\rightarrow}\left|\mathbf{v}_{i}\right|^{2}-\frac{1}{2} \sum_{i}^{\leftarrow}\left|\mathbf{v}_{i}\right|^{2}
\end{array}\right)
$$

where $\sum_{i} \overrightarrow{\text { and }} \sum_{i}^{\leftarrow}$ are sums over particles crossing the interface from left-toright and right-to-left, respectively.

This update effectively replaces the continuum flux component of the update to $U_{j}^{n+1}$ on edge $j+1 / 2$ by the flux of particles with their associated momentum through the edge. An analogous refluxing step occurs in the cell adjacent to the right-hand boundary of the particle region. Finally, note that this synchronization procedure guarantees conservation. The technical details of refluxing in higher dimensions (e.g., the treatment of corners) are discussed in Garcia et al. [4]. 


\section{Numerical results}

This section presents a series of computational examples, of progressively increasing sophistication, that demonstrate the accuracy and effectiveness of the algorithm refinement hybrid. First we examine an equilibrium system, then several non-equilibrium examples, concluding with a demonstration of adaptive refinement.

In our testing we compare three numerical schemes: the stochastic scheme based on three-stage Runge-Kutta for the Landau-Lifshitz Navier-Stokes equations discussed in Section 2.1 (Stoch. PDE only), and two algorithm refinement hybrids as described in Section 3. The first hybrid couples DSMC and stochastic RK3 (Stoch. Hybrid). The second hybrid is similar but without a stochastic flux in the LLNS equations, that is, using a deterministic version of RK3 (Deter. Hybrid). In some of the tests the results from these schemes are compared with data from a pure DSMC calculation.

In principle, the continuum grid of an AMAR hybrid may have as many hierarchical levels as necessary, and there may be many disjoint and/or linked DSMC patches. For simplicity, here we will consider a single DSMC region embedded within a single-level continuum grid. Furthermore, in the following numerical examples we use equal mesh spacing, $\Delta x$, and time step size, $\Delta t$, in both the continuum and particle methods. The straightforward adjustments necessary for implementing a DSMC grid with smaller $\Delta x$ and $\Delta t$ are presented in Section 3.

\subsection{Equilibrium system: state variables}

First, we consider a system in a periodic domain with zero bulk flow and uniform mean energy and mass density. Parameters for this equilibrium system are given in Table 1. Results from this first test problem are depicted in Figures 2-5. For both algorithm refinement hybrids, the particle patch is fixed at the center of the domain, covering cells $15-24$, indicated in the figures by vertical dotted black lines. For this equilibrium problem the particles in the patches used to provide boundary reservoirs for DSMC have velocities generated from the 
Table 1: System parameters (in cgs units) for simulations of a dilute gas at equilibrium in a periodic domain.

\begin{tabular}{|l|c|}
\hline Molecular diameter (argon) & $3.66 \times 10^{-8}$ \\
Molecular mass (argon) & $6.63 \times 10^{-23}$ \\
Reference mass density & $1.78 \times 10^{-3}$ \\
Reference temperature & 273 \\
Specific heat $\left(c_{v}\right)$ & $3.12 \times 10^{6}$ \\
Sound speed $(c)$ & 30781 \\
Reference mean free path $(\lambda)$ & $6.26 \times 10^{-6}$ \\
Reference mean free time $\left(t_{m}\right)$ & $1.64 \times 10^{-10}$ \\
System length & $1.25 \times 10^{-4}$ \\
System volume & $1.96 \times 10^{-16}$ \\
Number of computational cells & 40 \\
Cell length $(\Delta x)$ & $3.13 \times 10^{-6}$ \\
Time step $(\Delta t)$ & $1.0 \times 10^{-12}$ \\
\hline
\end{tabular}

Maxwell-Boltzmann distribution. In each simulation the system is initialized near the final state and allowed to relax for $5 \times 10^{6}$ time steps. Statistics are then gathered over $10^{7}$ time steps. Note that in these first tests we confirmed that all three schemes conserve total density, momentum and energy; recall that the hybrids are conservative due to the "refluxing" step. ${ }^{1}$

First, we examine mass density; results from the various numerical schemes are shown in Fig. 2. The first panel shows the mean of mass density at each spatial location, $\left\langle\rho_{i}\right\rangle$, and the second panel shows the variance, $\left\langle\delta \rho_{i}^{2}\right\rangle=\left\langle\left(\rho_{i}-\left\langle\rho_{i}\right\rangle\right)^{2}\right\rangle$. The third panel shows the center-point correlation, $\left\langle\delta \rho_{i} \delta \rho_{j=20}\right\rangle$, that is, the covariance of $\delta \rho_{i}$ with the value at the center of the

\footnotetext{
${ }^{1}$ When the grids move dynamically this exact conservation is lost because of quantization effects associated with initialization of a particle distribution from continuum data.
} 

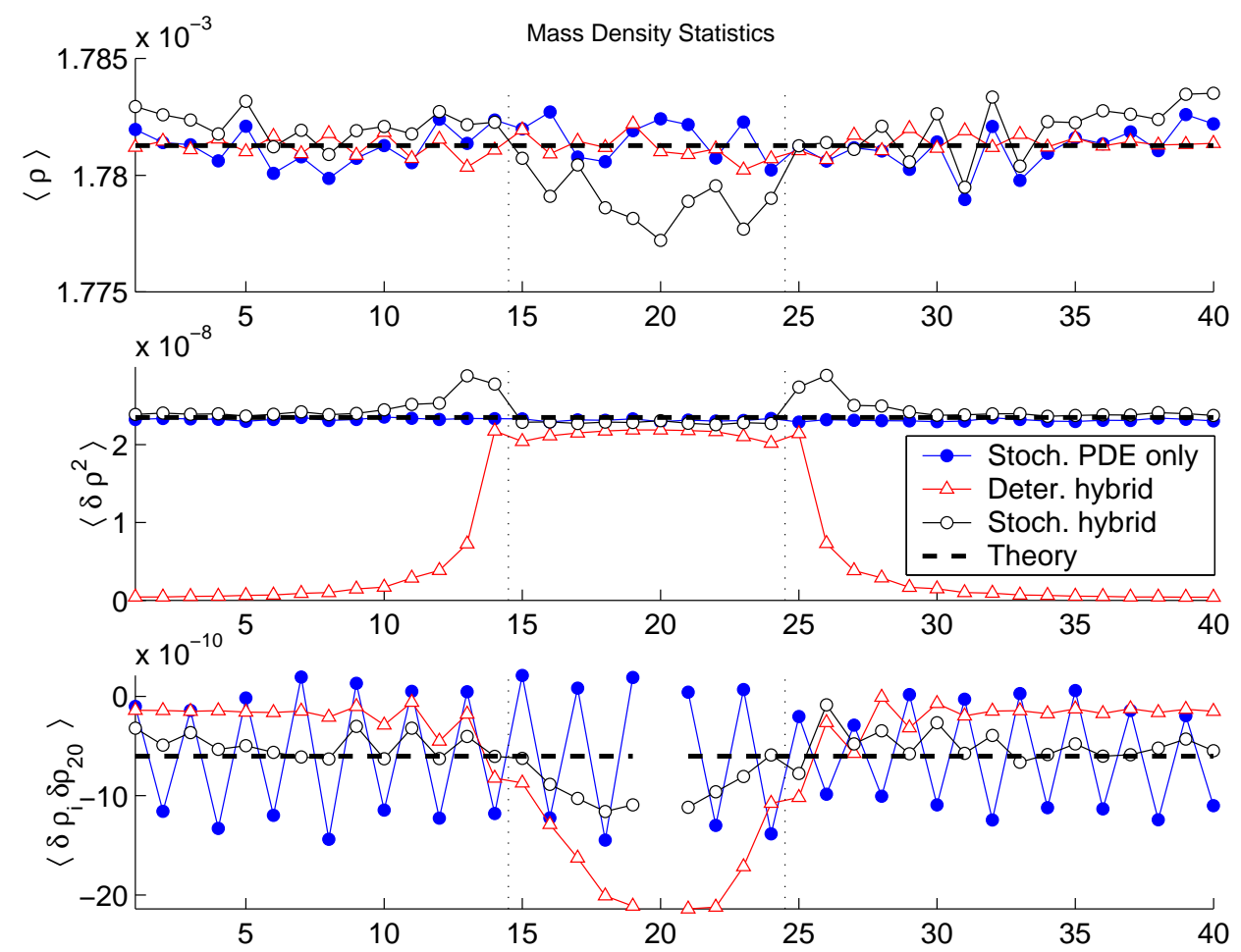

Figure 2: Mean, variance, and center point correlation of mass density versus spatial location for a system at equilibrium. Vertical dotted lines depict the boundaries of the particle region, for both hybrids. Note that, for clarity, the correlation value at $i=j=20$ is omitted from the plot.

domain $(j=20)$. These three quantities are estimated from samples as

$$
\begin{aligned}
\left\langle\rho_{i}\right\rangle & =\frac{1}{N_{s}} \sum_{n=1}^{N_{s}} \rho_{i}^{n}, \\
\left\langle\delta \rho_{i}^{2}\right\rangle & =\left(\frac{1}{N_{s}} \sum_{n=1}^{N_{s}}\left(\rho_{i}^{n}\right)^{2}\right)-\left\langle\rho_{i}\right\rangle^{2}, \\
\left\langle\delta \rho_{i} \delta \rho_{20}\right\rangle & =\left(\frac{1}{N_{s}} \sum_{n=1}^{N_{s}} \rho_{i}^{n} \rho_{20}^{n}\right)-\left\langle\rho_{i}\right\rangle\left\langle\rho_{20}\right\rangle,
\end{aligned}
$$

where $N_{s}=10^{7}$ is the number of samples and $i=1, \ldots, 40$. Similar statistics for x-momentum, y-momentum, and energy are displayed in Figs. 3 and 5 ; the statistics for z-momentum are similar to those for $\mathrm{y}$-momentum, and are omitted here. We only consider these conserved mechanical variables because the continuum scheme is based on them, they are easily measured in molecular simulations, and hydrodynamic variables, such as pressure and temperature, are directly obtained from these mechanical variables [36]. 

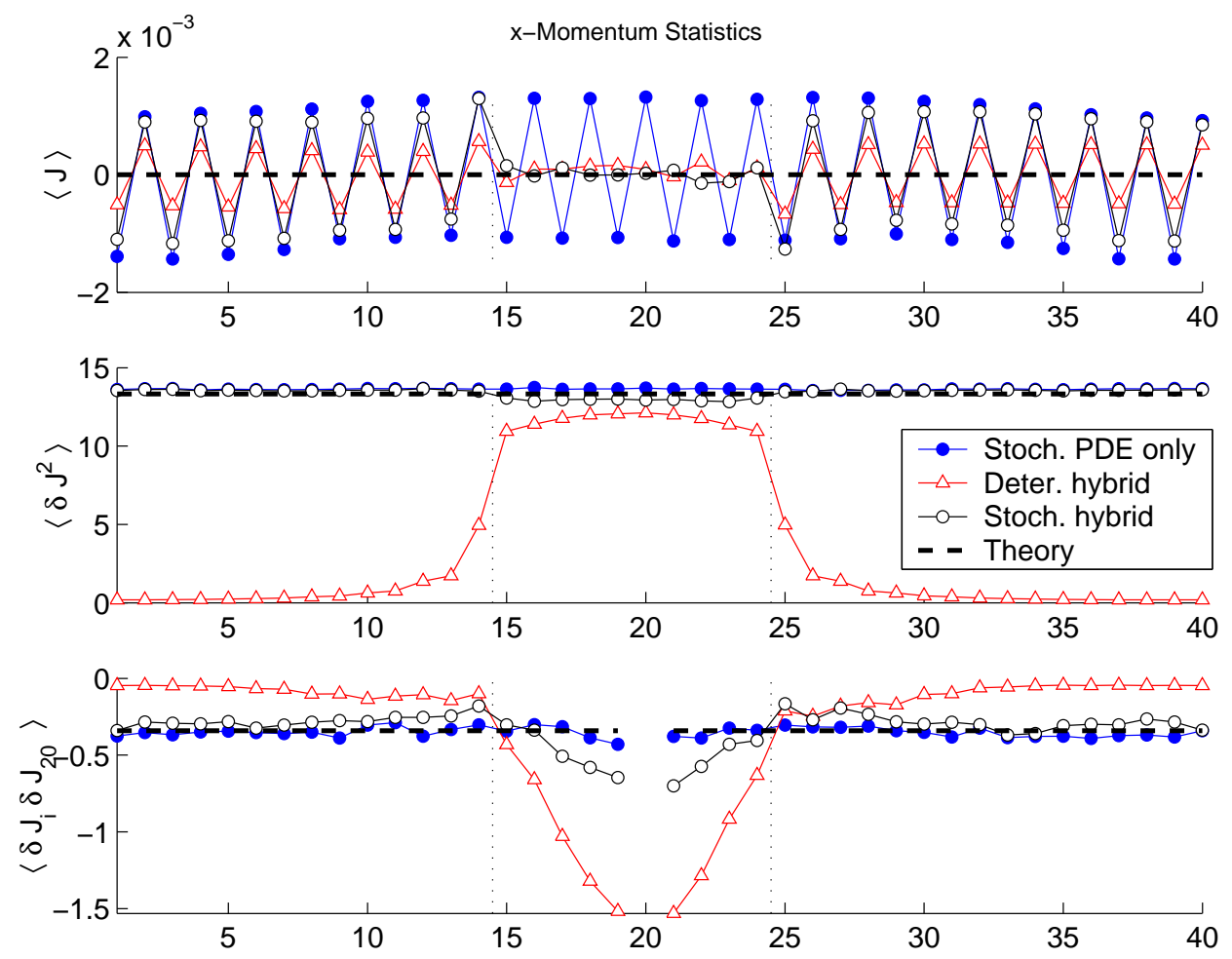

Figure 3: Mean, variance, and center point correlation of x-momentum versus spatial location for a system at equilibrium. Vertical dotted lines depict the boundaries of the particle region, for both hybrids. Note that, for clarity, the correlation value at $i=j=20$ is omitted from the plot.

We obtain the correct mean values for all three schemes, with the continuum method exhibiting some numerical oscillations, most notably in the $\mathrm{x}$ momentum. For the most part, the correct variance values are also obtained by the two stochastic schemes. In fact, the stochastic continuum method used here was developed in [17] with the particular goal of correctly reproducing the variances of conserved quantities. Nevertheless, some localized errors in variance introduced by the stochastic hybrid algorithm are evident in these figures. At the left and right boundaries of the particle patch, there is a peak error in the variance of about $23 \%$ for mass density and $14 \%$ for energy. These discrepancies are discussed in detail in Section 4.2.

Figs. $2-5$ also illustrate the effect on fluctuations when the hybrid's continuum PDE scheme does not include a stochastic flux. Clearly, the variances drop to near zero inside the deterministic continuum regions, left and right of 

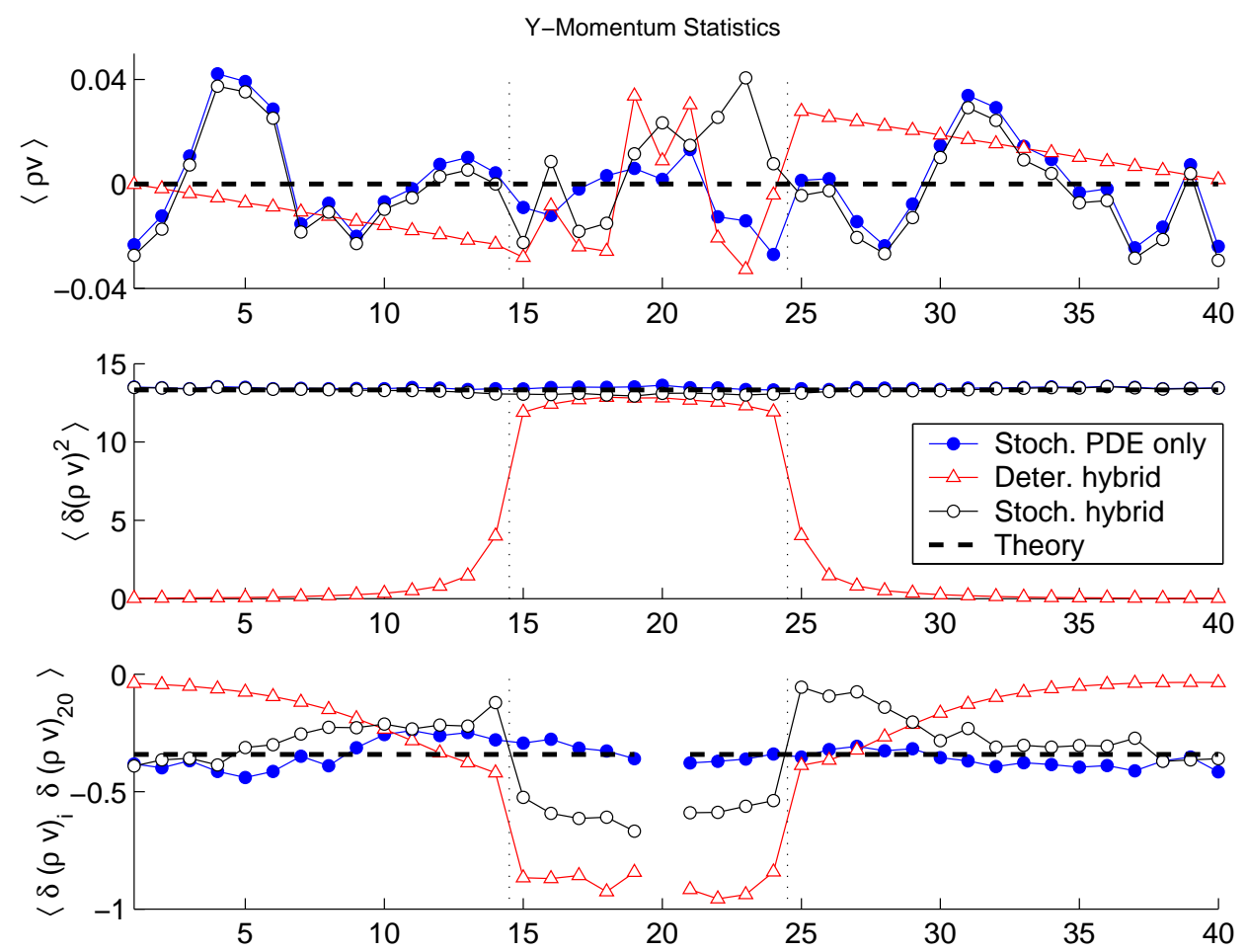

Figure 4: Mean, variance, and center point correlation of y-momentum versus spatial location for a system at equilibrium. Vertical dotted lines depict the boundaries of the particle region, for both hybrids. Note that, for clarity, the correlation value at $i=j=20$ is omitted from the plot.

the particle patch. More significantly, the variances within the patch are also damped. Even more interesting is the appearance of a large correlation of fluctuations in the particle region of the deterministic hybrid. It is well-known that correlations such as those appearing in the deterministic hybrid are present when a fluid is out of thermodynamic equilibrium (see Sec. 4.3). The results shown here for the center point correlation in the deterministic hybrid emphasize that the absence of fluctuations in the PDE causes the particle region to be in a non-equilibrium state; similar results were observed in $[11,12]$. This result underscores the importance of including fluctuations in the continuum model for problems in which the correct fluctuation structure is needed in the particle region. 

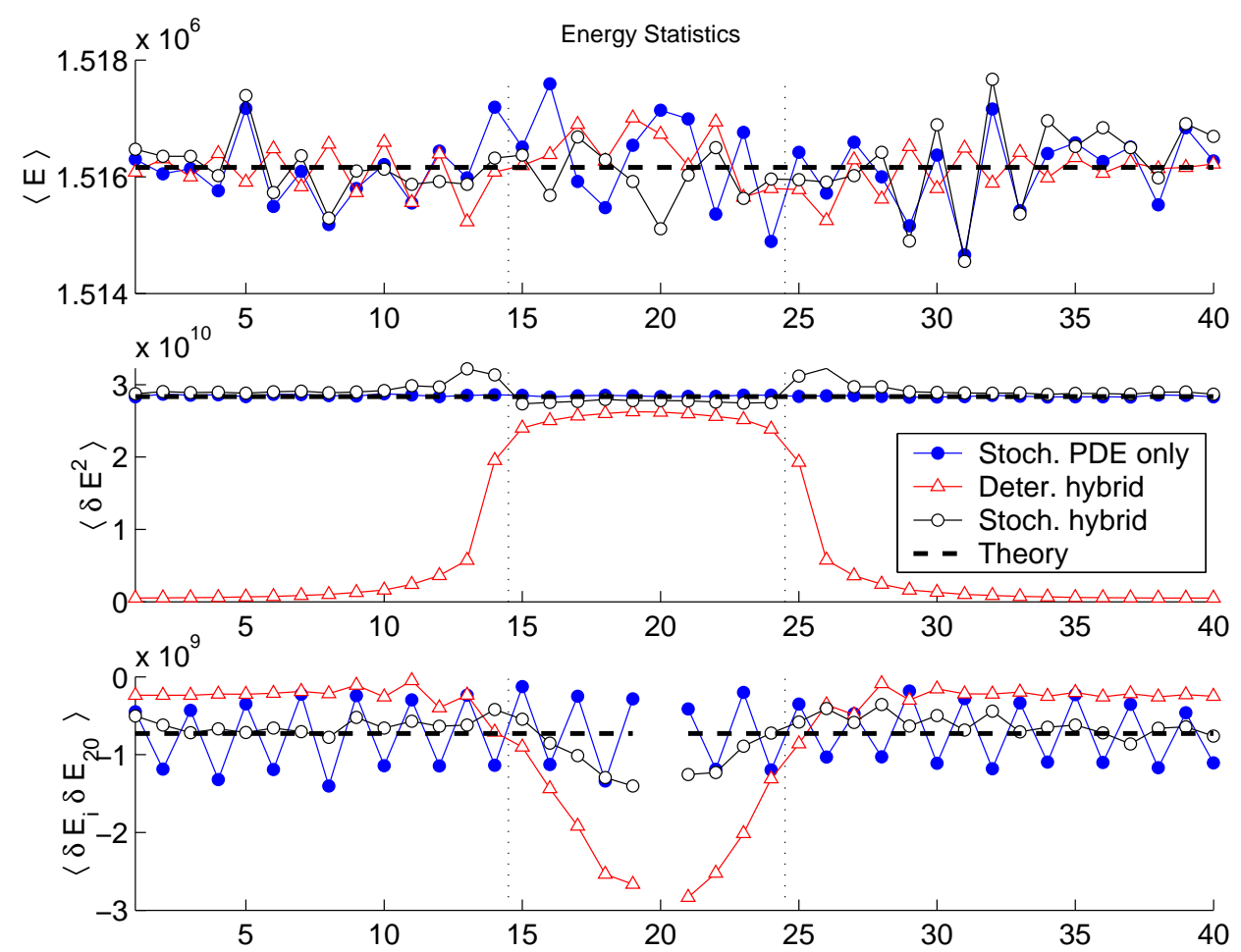

Figure 5: Mean, variance, and center point correlation of energy versus spatial location for a system at equilibrium. Vertical dotted lines depict the boundaries of the particle region, for both hybrids. Note that, for clarity, the correlation value at $i=j=20$ is omitted from the plot.

\subsection{Equilibrium system: fluxes}

Ideally, a hybrid method should produce a seamless integration at the interface between two algorithms. However, in Section 4.1 we saw that an error arises in the variance of mass density and the variance of energy at algorithm refinement interfaces where the particle method and continuum method interact (see Figs. 2 and 5). Fluxes are fundamental to the coupling mechanism in AMAR: continuum cells adjacent to a refinement interface are updated with particle flux (see Eq. (6)). Therefore, to investigate these errors in the variance, in this section we focus on statistical properties of the flux. We restrict our attention to mass flux, since it is determined by a single factor: $\mathrm{x}$-momentum in the continuum formulation, as in Eq. (2), and number of particle crossings in the discrete formulation, as in Eq. (7).

In [17] we demonstrated that the stochastic RK3 method and DSMC 
Table 2: Variance of x-momentum and of mass flux at equilibrium.

\begin{tabular}{|c|c|c|c|c|}
\hline$\left\langle\delta J^{2}\right\rangle$ & Computed value & Thermodynamic theory & & Pct. error \\
\hline Stoch. PDE only & 13.62 & 13.34 & & $2.1 \%$ \\
\hline DSMC & 13.21 & 13.34 & & $-1.0 \%$ \\
\hline$\overline{\left\langle\delta F^{(1)^{2}}\right\rangle}$ & Computed value & Hydrodynamic theory & Kinetic theory & Pct. error \\
\hline Stoch. PDE only & $2.84 \times 10^{-12}$ & $2.72 \times 10^{-12}$ & & $4.3 \%$ \\
\hline DSMC & $1.44 \times 10^{-10}$ & & $1.46 \times 10^{-10}$ & $-1.8 \%$ \\
\hline
\end{tabular}

both obtain the correct means, variances, and correlations, both spatial and temporal, of conserved quantities at equilibrium. Nevertheless, the nature of the fluxes differs markedly between the two methods. To illustrate this point, we consider a pure fluctuating continuum calculation and compare it to a pure DSMC calculation, for the same equilibrium test problem discussed in Section 4.1 .

As shown in Table 2, the variance of $\mathrm{x}$-momentum, $\left\langle\delta J^{2}\right\rangle$, as obtained by the continuum method and by DSMC, are each in agreement with thermodynamic theory. (Derivation of the theoretically exact variance is discussed in [36].) Hydrodynamic theory directly relates the mass flux to the momentum, and for the stochastic RK3 scheme the variance of mass flux is given by

$$
\left\langle\left(\delta F^{(1)}\right)^{2}\right\rangle=2\left(\frac{\Delta t}{\Delta x}\right)^{2}\left\langle\delta J^{2}\right\rangle=2\left(\frac{\Delta t}{\Delta x}\right)^{2} \frac{\rho k_{B} T}{\sigma \Delta x} .
$$

(See also the discussion of Eq. (3).) On the other hand, kinetic theory predicts that the number of particles crossing a cell interface is Poisson distributed, with

$$
\left\langle N^{\rightarrow}\right\rangle=\left\langle\left(\delta N^{\rightarrow}\right)^{2}\right\rangle=\frac{1}{2 \sqrt{\pi}}\left(\frac{\rho}{m}\right) \sigma \Delta t \sqrt{\frac{2 k_{B} T}{m}} .
$$

(Derivation is discussed in [31], for example.) From this we have the variance of the mass flux given by

$$
\begin{aligned}
\left\langle\left(\delta F^{(1)}\right)^{2}\right\rangle & =\frac{m^{2}}{(\sigma \Delta x)^{2}}\left\langle\delta\left(N^{\rightarrow}-N^{\leftarrow}\right)^{2}\right\rangle=\frac{2 m^{2}}{(\sigma \Delta x)^{2}}\left\langle\left(\delta N^{\rightarrow}\right)^{2}\right\rangle \\
& =\frac{m \rho}{\sqrt{\pi} \sigma} \frac{\Delta t}{\Delta x^{2}} \sqrt{\frac{2 k_{B} T}{m}} .
\end{aligned}
$$

Comparing eqns. (8) and (9) one finds that the hydrodynamic and kinetic theory expressions match when the Courant number, $C=c \Delta t / \Delta x$, is order one yet for the runs presented here $C \approx 10^{-2}$ (see Table 1 ). 
From Table 2, we see that the variance of the mass flux for the continuum method is in good agreement with the hydrodynamic theory, Eq. (8), and the corresponding DSMC result is in good agreement with kinetic theory, Eq. (9). Yet, the two variances of mass flux differ by over two orders of magnitude. To understand the nature of this discrepancy, we investigate the time correlation of the mass flux.

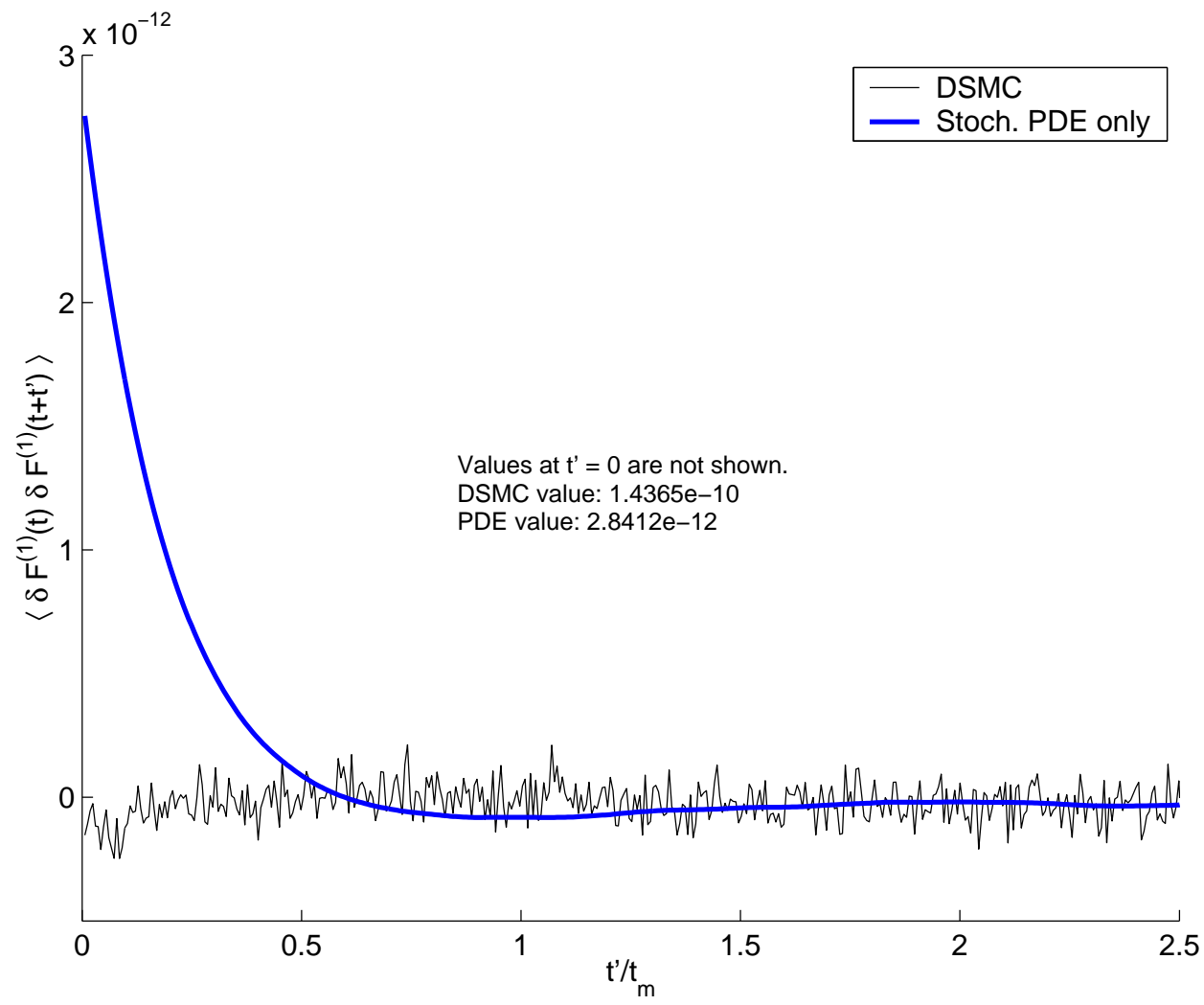

Figure 6: Time correlations of mass flux, for the particle method (DSMC) and the PDE method.

To estimate the time correlation of mass flux for a timeshift of $t^{\prime}$ we calculate $\left\langle\delta F^{(1)}(t) \delta F^{(1)}\left(t+t^{\prime}\right)\right\rangle$ in each of the 40 computational cells from approximately $10^{5}$ data samples. The average value of each time correlation over the 40 computational cells is displayed in Fig. 6, for stochastic RK3 and for DSMC. Time correlation data is displayed in units of mean free collision time $\left(t_{m}\right)$.

In Fig. 6 we see that the mass flux for DSMC decorrelates immediately, whereas the continuum mass flux decorrelates after approximately one half of 
one mean free collision time. Note that for all the simulation results presented here, the stochastic PDE and the DSMC use the same time step, and that time step is over two orders of magnitude smaller than $t_{m}$. The order of the discrepancy in Table 2 is now clear. The hydrodynamic formulation is only accurate at hydrodynamic time scales, that is, at time scales that are large compared to $t_{m}$. Further investigations (not presented here) indicate that when the two methods are run using a significantly larger time step, the variance and time correlations of the mass flux are in general agreement between the two methods. However, at large time step, the truncation error for the PDE scheme negatively effects the results for other quantities, e.g., the variance in conserved quantities. Given that the statistical properties of the fluxes differ between hydrodynamic scales and molecular (kinetic) scales, it is not surprising that the variances of conserved quantities are not seamless at the interface of the two methods. Why this effect is most prominent for density and energy variances is still under investigation. This issue is discussed further in the concluding section. 


\subsection{Non-equilibrium system: temperature gradient}

In the early 1980's, a variety of statistical mechanics calculations predicted that a fluid under a non-equilibrium constraint, such as a temperature gradient, would exhibit long-range correlations of fluctuations [37, 38]. Due to the asymmetry of sound waves moving parallel versus anti-parallel to the temperature gradient, quantities that are independent at equilibrium, such as density and momentum fluctuations, also have long-range correlations. These predictions were qualitatively confirmed by light scattering experiments [39], yet the effects are subtle and difficult to measure accurately in the laboratory. Molecular simulations confirm the predicted correlations of non-equilibrium fluctuations for a fluid subjected to a temperature gradient $[40,25]$ and to a shear [41]. With these predictions in mind, we consider a system with a temperature gradient. Specifically, the boundary conditions are thermal walls at $273 \mathrm{~K}$ and $819 \mathrm{~K}$; the other system parameters are as shown in Table 1. This non-equilibrium state is extreme, with a temperature gradient of millions of degrees per centimeter, yet it is accurately modeled by DSMC, which was originally developed to simulate strong shock waves.

The system is initialized near the final state and is allowed to relax for $10^{6}$ time steps before samples are taken at each computational cell over $10^{8}$ time steps. The measure shown here (Figures 7-9) is the spatial correlation between mass density and momentum, specifically $\left\langle\delta \rho_{i} \delta J_{20}\right\rangle$. A pure DSMC simulation is used as the benchmark.

The stochastic hybrid method outperforms the pure continuum method in this non-equilibrium test case. Although the stochastic RK3 method gives a good match to the DSMC benchmark away from the correlation point, the results deteriorate near the correlation point (Fig. 7). In the stochastic hybrid method, a particle patch is placed around the region of difficulty and the results are significantly improved (Fig. 8). Finally, in Fig. 9 we consider the hybrid that couples deterministic RK3 with DSMC. Again, DSMC is employed in a single patch at the center of the domain. However, with fluctuations suppressed in the remainder of the domain, the overall results suffer. Strikingly, the results suffer not only in the continuum region, but also within the particle region. 


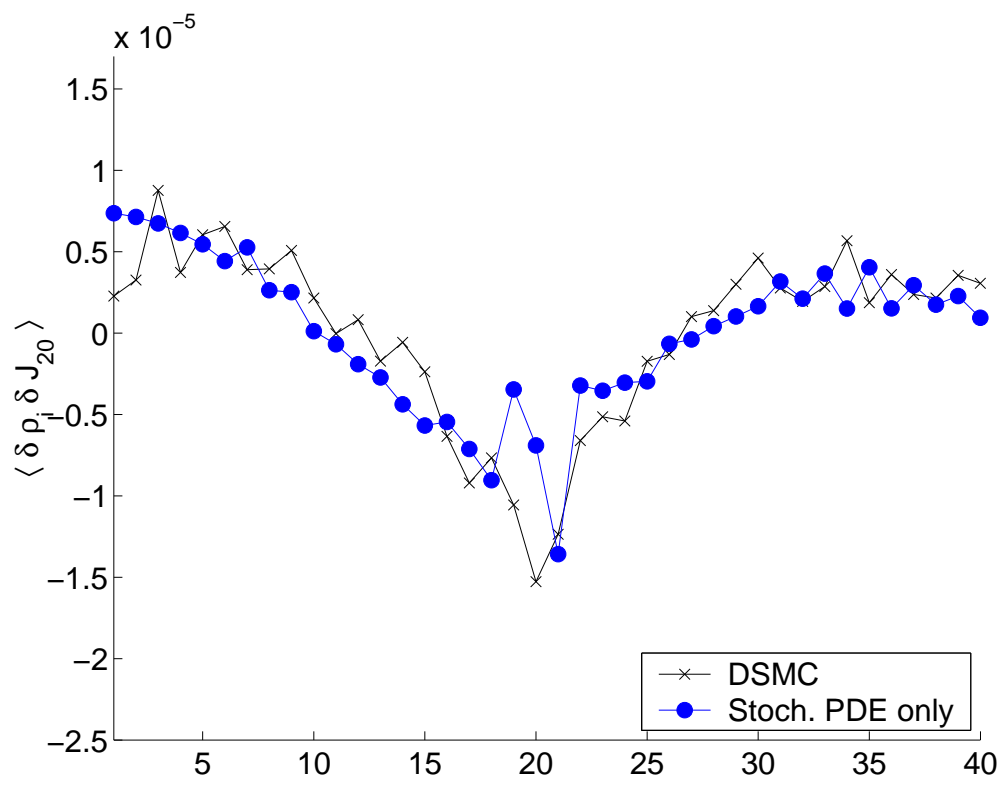

Figure 7: Center point correlation of mass density and x-momentum for a system under a steep temperature gradient.

\subsection{Non-equilibrium system: strong moving shock}

In this time-dependent problem, we consider a Mach 2 shock traveling through a domain that includes a static refinement region. The objective of this example is to test how well the hybrid performs when a strong nonlinear wave crosses the interface between continuum and particle regions. Dirichlet boundary conditions are used at the domain boundaries; values for the left-hand (LHS) and righthand (RHS) states are given in Table 3.

We observe a relatively local and short-lived error that indicates an impedance mismatch between the continuum and particle regions, as shown in Figure 10. The mass density profile depicted by the dark line is an average profile from an ensemble of 2000 stochastic hybrid runs. Results from an ensemble of 2000 pure stochastic PDE simulations of the traveling wave, without a particle patch, are shown for comparison. The first panel of Fig. 10 also includes the mass density profile from a single stochastic hybrid simulation, illustrating the relative magnitude of the thermal fluctuations.

At time $t_{0}$, before the shock enters the particle region, the ensemble- 


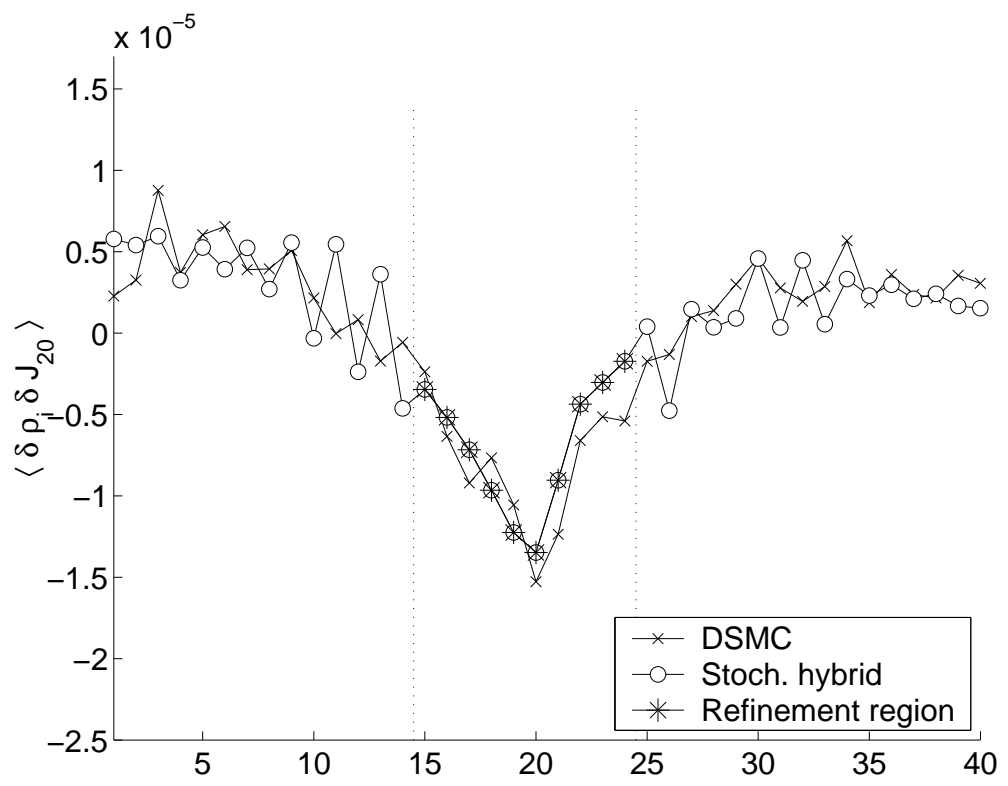

Figure 8: Center point correlation of mass density and x-momentum for a system under a steep temperature gradient. Vertical dotted lines depict the boundaries of the particle region for the hybrid method.

averaged data is smooth. At time $t_{1}$, a spurious reflected wave is formed at the interface on the left-hand side of the particle patch. This spurious acoustic wave is damped as it propagates leftward, vanishing by time $t_{4}$. Another small error effect is seen as the shock exits the particle patch, at time $t_{5}$, but it is barely discernible by time $t_{7}$. This example demonstrates the robustness and stability of the treatment of the interface between the particle region and the continuum solver.

A well-known feature of CFD solvers is the artificial steepening of viscous shock profiles; it is also well-established that DSMC predicts shock profiles accurately $[31,42]$. At times $t_{2}$ through $t_{6}$, we see a steepness discrepancy between the ensemble hybrid profile and the ensemble PDE-only profile. Within the particle patch, the DSMC algorithm correctly resolves a more shallow profile.

In this example, the Chapman-Enskog distribution was used to initialize velocities of particles that enter the refinement region from the continuum 


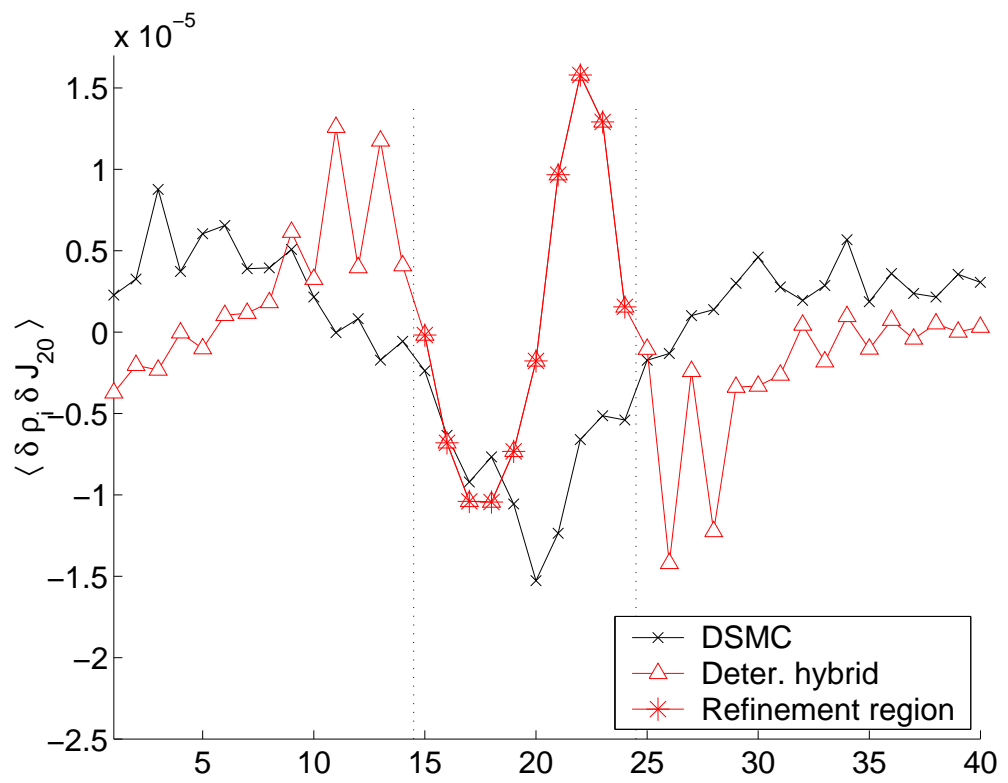

Figure 9: Center point correlation of mass density and x-momentum for a system under a steep temperature gradient. Vertical dotted lines depict the boundaries of the particle region for the hybrid method.

region. This approach was found to result in a somewhat reduced impedance mismatch compared to the Maxwell-Boltzmann distribution. 
Table 3: System parameters (in cgs units) for simulations of a traveling shock.

\begin{tabular}{|l|c|c|}
\hline Mach number & \multicolumn{2}{|c|}{2.0} \\
\hline & LHS & RHS \\
Mass density & $4.07 \times 10^{-3}$ & $1.78 \times 10^{-3}$ \\
Velocity (x-direction) & 34629 & 0 \\
Temperature & 567 & 273 \\
Sound speed & 44373 & 30781 \\
Mean free path & $2.74 \times 10^{-6}$ & $6.26 \times 10^{-6}$ \\
\hline Cell length $(\Delta x)$ & \multicolumn{2}{|c|}{$3.13 \times 10^{-6}$} \\
Time step $(\Delta t)$ & \multicolumn{2}{|c|}{$1.0 \times 10^{-12}$} \\
\hline
\end{tabular}



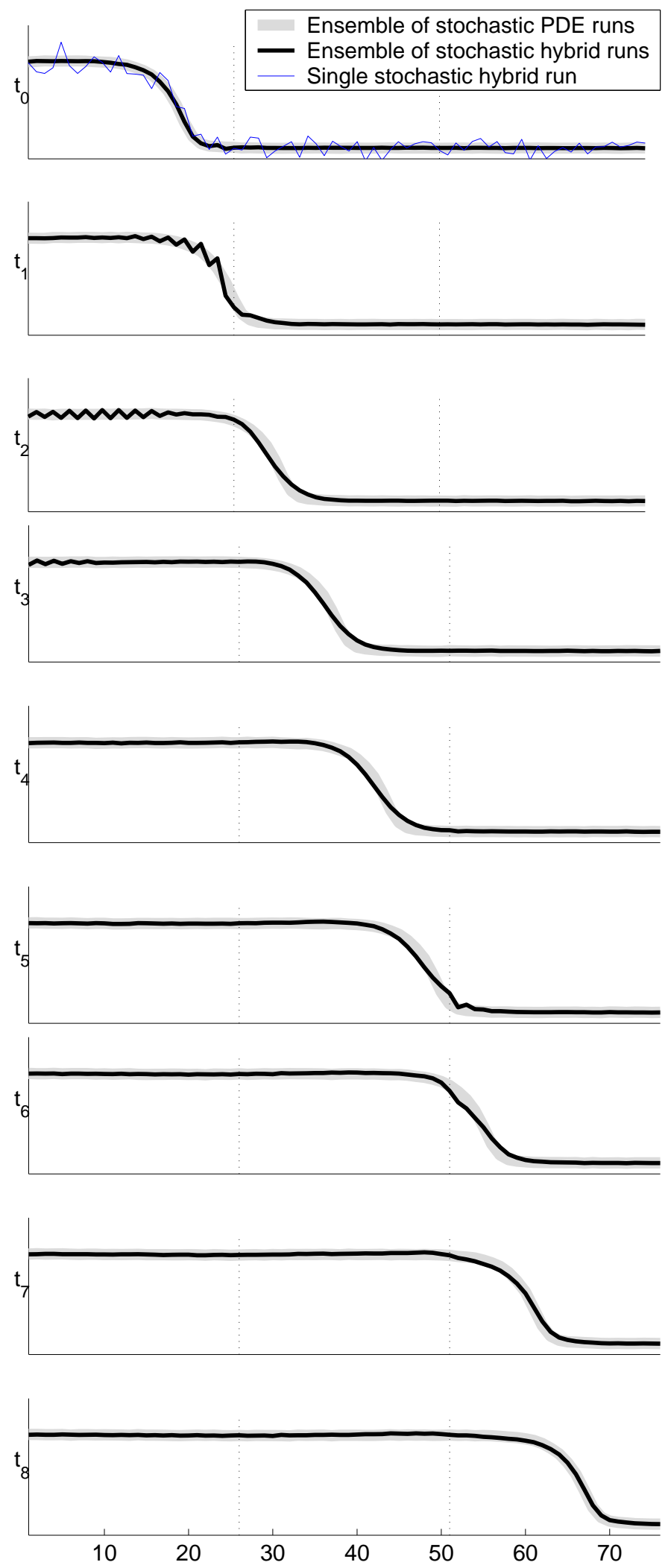

Figure 10: Mass density profiles for a viscous shock wave traveling through a fixed refinement region (indicated by vertical dotted lines). The time elapsed between each panel is $300 \Delta t$; see Table 3 for system parameters. 


\subsection{Adaptive refinement}

The final numerical test demonstrates the adaptive refinement capability of our hybrid algorithm. As in Section 4.4, a strong traveling shock (Mach 2) moves through a domain with Dirichlet boundary conditions. System parameters are given in Table 3 . Here, though, the location of a particle patch is determined dynamically by identifying cells in which the gradient of pressure exceeds a given tolerance; the particle patch is shown in Figure 11 by vertical dotted lines.

Since the fluctuations produce steep localized gradients nearly everywhere, a regional gradient measure, $D(P)$, is employed to detect large-scale gradients. Large scale gradients in pressure provide an effective criterion for identifying the presence of a shock wave. This is implemented as:

$$
D(P)_{j}=\frac{1}{S \Delta x}\left[\frac{1}{S} \sum_{i=1}^{S} P_{j+i}-\frac{1}{S} \sum_{i=1}^{S} P_{j-(i-1)}\right],
$$

where $S$ indicates the width of the gradient stencil (we use $S=6$ ). For an equilibrium system, the expected variance of $D(P)$ is estimated by

$$
\left\langle\delta D(P)^{2}\right\rangle=\frac{2}{S^{3} \Delta x^{2}}\left(\left\langle\delta \rho^{2}\right\rangle R^{2} T_{0}^{2}+\rho_{0}^{2} R^{2}\left\langle\delta T^{2}\right\rangle\right)=\frac{10}{3} \frac{1}{S^{3} N_{c}}\left(\frac{P_{0}}{\Delta x}\right)^{2}
$$

where $\rho_{0}, T_{0}$ and $P_{0}$ are the reference mass density, temperature and pressure for the system and $N_{c}$ is the number of particles in a cell at reference conditions. (This variance can be found using the ideal gas law and expressions derived in [36].) Note that using a wide stencil limits the variation even when $N_{c}$ is small (and, consequently, fluctuations are large).We select cells $j$ for refinement where $D(P)_{j}$ exceeds the equilibrium value, namely zero, by three standard deviations. The resulting particle patch is extended by a buffer of four cells on each side.

In this implementation, we re-evaluate the location of the particle patch every 100 time steps. When the extent of the refinement region changes, some continuum cells may be added to the DSMC patch, some DSMC cells may become continuum cells, and some DSMC cells may remain in the refinement patch. For continuum cells that are added to the DSMC patch, particles are initialized from the underlying continuum data, as in the case of a static patch. For DSMC cells that should no longer be included in the refinement patch, particle data is averaged onto the continuum grid, as in Eqs. (5), then discarded. 
For those DSMC cells that remain in the particle patch, the particle data is retained.
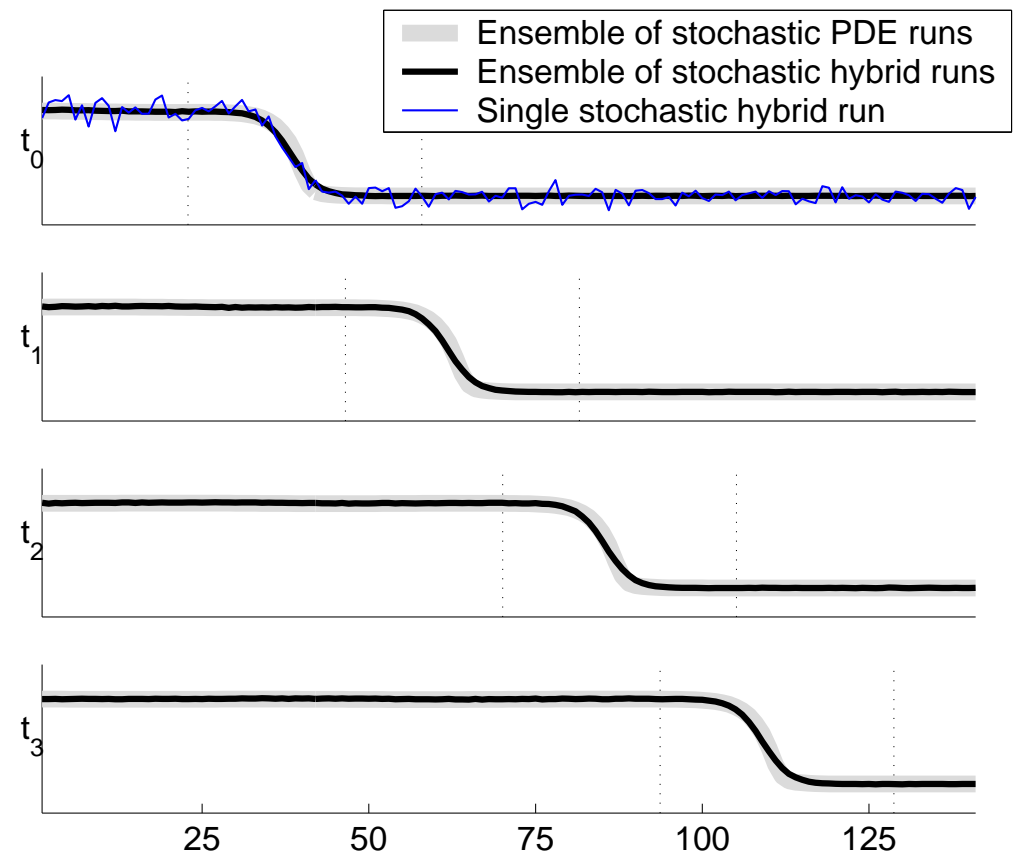

Figure 11: Mass density profiles for a viscous shock wave, demonstrating adaptive mesh refinement: the refinement region, indicated by vertical dotted lines, is determined dynamically at runtime. The time elapsed between each panel is $1200 \Delta t$; see Table 3 for system parameters.

The mass density profile depicted by the dark line is an average profile from an ensemble of 2000 stochastic hybrid runs. The first panel of Fig. 11 also includes the mass density profile from a single stochastic hybrid simulation, illustrating the relative magnitude of the thermal fluctuations. Results from an ensemble of 2000 pure stochastic PDE simulations of the traveling wave, without a particle patch, are also shown for comparison. As in Figure 10, we that a more shallow profile is captured by the DSMC representation of the viscous shock (i.e. by the hybrid that uses DSMC in the vicinity of the shock) versus the artificially steep profile produced by the PDE-only system. 


\section{Conclusions and Further Work}

We have constructed a hybrid algorithm that couples a DSMC molecular simulation with a new numerical solver for the Landau-Lifshitz Navier-Stokes equations for fluctuating compressible flow. The algorithm allows the particle method to be used locally to approximate the solution while modeling the system using the mean field equations in the remainder of the domain. In tests of the method we have demonstrated that it is necessary to include the effect of fluctuations, represented as a stochastic flux, in the mean field equations to ensure that the hybrid preserved key properties of the system. As expected, not representing fluctuations in the continuum regime leads to a decay in the variance of the solution that penetrates into the particle region. Somewhat more surprising is that the failure to include fluctuations was shown to introduce spurious correlations of fluctuations in equilibrium simulations and for rarefactions.

There are several directions that we plan to pursue in future work. As a first step, we plan to extend the methodology to two and three dimensional hybrids. The key algorithmic steps developed here extend naturally to multiple dimensions. For more general applications, an overall approach needs to be implemented to support particle regions defined by a union of non-overlapping patches. Another area of development is to include additional physical effects in both the continuum and particle models. As a first step in this direction, is it straightforward to include the capability to model different species. This provides the necessary functionality needed to study Rayleigh-Taylor instabilities and other mixing phenomena. A longer term goal along these lines would be to include chemical reactions within the model to enable the study of ignition

phenomena. Finally, we note that the results presented here suggest a number of potential improvements to the core methodology. Of particular interest in this area would be approaches to the fluctuating continuum equations that can accurately capture fluctuations while taking a larger time step. This would not only improve the efficiency of the methodology, it would also enable the continuum solver to take time steps at hydrodynamic time scales which should serve to improve the quality of coupling between continnum and particle regions. 


\section{Acknowledgement}

This work was supported, in part, by the Applied Mathematics Program of the DOE Office of Mathematics, Information, and Computational Sciences under the U.S. Department of Energy under contract No. DE-AC02-05CH11231.

\section{Appendix: Random placement of particles with a density gradient}

Consider the problem of selecting a random position for a particle within a rectangular cell. The density in the cell varies linearly with $\rho_{0}$ being the density at the center (which is also the mean density). For a cell with dimensions $\Delta x$, $\Delta y$, and $\Delta z$, taking the origin at the corner of the cell we have

$$
\rho(x, y, z)=\rho_{0}+a_{x}(x-\Delta x / 2)+a_{y}(y-\Delta y / 2)+a_{z}(z-\Delta z / 2)
$$

where $a_{x}=\partial \rho / \partial x$. The probability that a particle has position component $x$ is

$$
P(x)=\frac{\int_{0}^{\Delta y} d y \int_{0}^{\Delta z} d z \rho(x, y, z)}{\rho_{0} \Delta x \Delta y \Delta z}=\frac{1+\gamma_{x}\left(x / \Delta x-\frac{1}{2}\right)}{\Delta x}
$$

where $\gamma_{x} \equiv \Delta x a_{x} / \rho_{0}$. It will be more convenient to work in the dimensionless variable $X=x / \Delta x$. Since $P(x) d x=P(X) d X$,

$$
P(X)=1+\gamma_{x}\left(X-\frac{1}{2}\right) .
$$

By the method of inversion [30] one may generate random values from this distribution by

$$
X=\gamma_{x}^{-1}\left[\left(\gamma_{x} / 2-1\right)+\left[\left(\gamma_{x} / 2-1\right)^{2}+2 \gamma_{x} \mathcal{R}\right]^{1 / 2}\right]
$$

where $\mathcal{R}$ is a random value uniformly distributed in $[0,1]$. The reader is cautioned that the above is susceptible to round-off error for $\gamma_{x} \approx 0$ (i.e., small gradient case). Note that in that limit,

$$
X \approx \frac{\mathcal{R}}{1-\gamma_{x} / 2}
$$

from which we recover the expected result that $X=\mathcal{R}$ when $\gamma_{x}=0$. 
The selection of the $y$ component of the position is complicated by the fact that it is not independent of the $x$ component. The conditional probability of the $y$ component of position is

$$
P(y \mid x)=\frac{P(x, y)}{P(x)}=\frac{1}{\Delta y}\left[1+\frac{\Delta y a_{y} / \rho_{0}}{1+\left(\Delta x a_{x} / \rho_{0}\right)\left(x / \Delta x-\frac{1}{2}\right)}\left(\frac{y}{\Delta y}-\frac{1}{2}\right)\right] .
$$

Define $\gamma_{y} \equiv \Delta y a_{y} / \rho_{0}$ and $Y \equiv y / \Delta y$, then

$$
P(Y \mid X)=1+\frac{\gamma_{y}}{P(X)}\left(Y-\frac{1}{2}\right)
$$

Fortunately, after selecting $X$ the selection of $Y$ is simple; $Y$ is generated in the same way as $X$ but with $\gamma_{y} / P(X)$ in the place of $\gamma_{x}$.

Finally, to select the $z$ component of position the procedure is similar with

$$
P(Z \mid X, Y)=1+\frac{\gamma_{z}}{P(X, Y)}\left(Z-\frac{1}{2}\right)
$$

where $P(X, Y)=P(X \mid Y) P(Y)=1+\gamma_{x}\left(X-\frac{1}{2}\right)+\gamma_{y}\left(Y-\frac{1}{2}\right)$. Again, the $z$ component can be generate as the $x$ component with $\gamma_{z} / P(X, Y)$ replacing $\gamma_{x}$.

\section{References}

[1] M. Berger and J. Oliger. Adaptive mesh refinement for hyperbolic partial differential equations. J. Comput. Phys., 53:484, 1984.

[2] M. Berger and P. Colella. Local adaptive mesh refinement for shock hydrodynamics. J. Comput. Phys., 82:64, 1989.

[3] J.B. Bell, M. Berger, J. Saltzman, and M. Welcome. Three-dimensional adaptive mesh refinement for hyperbolic conservation law. SIAM J. Sci. Comput., 15:127, 1994.

[4] A.L. Garcia, J.B. Bell, W.Y. Crutchfield, and B.J. Alder. Adaptive mesh and algorithm refinement using Direct Simulation Monte Carlo. J. Comput. Phys., 154(1):134-155, 1999.

[5] Kai Kadau, Timothy C. Germann, Nicolas G. Hadjiconstantinou, Peter S. Lomdahl, Guy Dimonte, Brad Lee Holian, and Berni J. Alder. Nanohydrodynamics simulations: An atomistic view of the Rayleigh-Taylor instability. PNAS, 101(16):5851-5855, 2004. 
[6] K. Kadau, C. Rosenblatt, J.L. Barber, T.C. Germann, Z. Huang, P. Carls, and B.J. Alder. The importance of fluctuations in fluid mixing. PNAS, (To Appear), 2007.

[7] H.S. Wijesinghe and N.G. Hadjiconstantinou. Discussion of hybrid atomistic-continuum methods for multiscale hydrodynamics. International Journal for Multiscale Computational Engineering, 2(2):189-202, 2004.

[8] N. G. Hadjiconstantinou. Discussion of recent developments in hybrid atomistic-continuum methods for multiscale hydrodynamics. Bulletin of the Polish Academy of Sciences, 53(4):335-342, 2005.

[9] H.S. Wijesinghe, R. Hornung, A.L. Garcia, and N.G. Hadjiconstantinou. Three-dimensional hybrid continuum-atomistic simulations for multiscale hydrodynamics. J. Fluids Eng., 126:768-777, 2004.

[10] F.J. Alexander, A.L. Garcia, and D.M. Tartakovsky. Algorithm refinement for stochastic partial differential equations: I. Linear diffusion. J. Comput. Phys., 182(1):47-66, 2002.

[11] F.J. Alexander, A.L. Garcia, and D.M. Tartakovsky. Algorithm refinement for stochastic partial differential equations: II. Correlated systems. J. of Comp. Phys., 207:769-787, 2005.

[12] J.B. Bell, J. Foo, and A. Garcia. Algorithm refinement for the stochastic Burgers' equation. J. Comp. Phys., 223:451-468, 2007.

[13] T.E. Schwarzentruber, L.C. Scalabrin, and I.D. Boyd. A modular particlecontinuum numerical method for hypersonic non-equilibrium gas flows. Journal of Computational Physics, 2007.

[14] G. Giupponi, G. De Fabritiis, and P. V. Coveney. Hybrid method coupling fluctuating hydrodynamics and molecular dynamics for the simulation of macromolecules. Journal of Chemical Physics, 126:154903, 2007.

[15] V.I. Kolobov, R.R. Arslanbekov, V.V. Aristov, A.A. Frolova, and S.A. Zabelok. Unified solver for rarefied and continuum flows with adaptive mesh 
and algorithm refinement. Journal of Computational Physics, 223:589-608, 2007.

[16] R. Delgado-Buscalioni and P. V. Coveney. Continuum-particle hybrid coupling for mass, momentum, and energby transfers in unsteady fluid flow. Physical Review E (Statistical, Nonlinear, and Soft Matter Physics), 67(4):046704, 2003.

[17] J.B. Bell, A.L. Garcia, and S.A. Williams. Numerical methods for the stochastic Landau-Lifshitz Navier-Stokes equations. in process, 2007.

[18] L.D. Landau and E.M. Lifshitz. Fluid Mechanics, volume 6 of Course of Theoretical Physics. Pergamon, 1959.

[19] M. Bixon and R. Zwanzig. Boltzmann-Langevin equation and hydrodynamic fluctuations. Phys. Rev., 187(1):267-272, Nov 1969.

[20] R. F. Fox and G. E. Uhlenbeck. Contributions to non-equilibrium thermodynamics. I. Theory of hydrodynamical fluctuations. Phys. Fluids, 13(8):1893-1902, 1970.

[21] G.E. Kelly and M.B. Lewis. Hydrodynamic fluctuations. Physics of Fluids, 14(9):1925-1931, 1971.

[22] E. Calzetta. Relativistic fluctuating hydrodynamics. Class. Quantum Grav., 15:653, 1998.

[23] P. Espa nol. Stochastic differential equations for non-linear hydrodynamics. Physica A, 248:77, 1998.

[24] A.L. Garcia and C. Penland. Fluctuating hydrodynamics and principal oscillation pattern analysis. J. Stat. Phys., 64(5-6):1121-1132, 1991.

[25] M. Malek-Mansour, A.L. Garcia, G.C. Lie, and E. Clementi. Fluctuating hydrodynamics in a dilute gas. Phys. Rev. Lett., 58:874-877, 1987.

[26] M. Mareschal, M. Malek-Mansour, G. Sonnino, and E. Kestemont. Dynamic structure factor in a nonequilibrium fluid: A molecular-dynamics approach. Phys. Rev. A, 45:7180-7183, May 1992. 
[27] S. Gottleib and C. Shu. Total variation diminishing Runge-Kutta schemes. Mathematics of Computation, 67(221):73-85, 1998.

[28] J. Qiu and C. Shu. Runge-Kutta discontinuous Galerkin method using WENO limiters. SIAM Journal on Scientific Computing, 26(3):907-929, 2005 .

[29] F.J. Alexander and A.L. Garcia. The Direct Simulation Monte Carlo method. Computers in Physics, 11(6):588-593, 1997.

[30] A.L. Garcia. Numerical Methods for Physics. Prentice Hall, 2nd edition, 2000.

[31] G.A. Bird. Molecular Gas Dynamics and the Direct Simulation of Gas Flows. Clarendon, Oxford, 1994.

[32] W. Wagner. A convergence proof for Bird's Direct Simulation Monte Carlo method for the Boltzmann equation. J. Stat. Phys., 66:1011, 1992.

[33] A.L. Garcia, M. Malek-Mansour, G. Lie, and E. Clementi. Numerical integration of the fluctuating hydrodynamic equations. J. Stat. Phys., 47:209, 1987.

[34] M. Malek-Mansour, A.L. Garcia, J.W. Turner, and M. Mareschal. On the scattering function of simple fluids in finite systems. J. Stat. Phys., 52:295, 1988.

[35] A.L. Garcia and B.J. Alder. Generation of the Chapman-Enskog distribution. J. of Comp. Phys., 140:66-70, 1998.

[36] A.L. Garcia. Estimating hydrodynamic quantities in the presence of fluctuations. Comm. App. Math. and Comp. Sci., 1(1):53-78, 2006.

[37] R. Schmitz. Fluctuations in nonequilibrium fluids. Physics Reports, 171:1, 1988.

[38] J. M. Ortiz de Zarate and J. V. Sengers. Hydrodynamic Fluctuations in Fluids and Fluid Mixtures. Elsevier Science, 2007. 
[39] D. Beysens, Y. Garrabos, and G. Zalczer. Experimental evidence for Brillouin asymmetry induced by a temperature gradient. Phys. Rev. Lett., 45:403, 1980.

[40] A.L. Garcia. Nonequilibrium fluctuations studied by a rarefied gas simulation. Phys. Rev. A, 34:1454, 1986.

[41] A.L. Garcia, M. Malek-Mansour, G.C. Lie, M. Mareschal, and E. Clementi. Hydrodynamic fluctuations in a dilute gas under shear. Phys. Rev. A, $36: 4348-4355,1987$.

[42] H. Alsmeyer. Density profiles in argon and nitrogen shock waves measured by the absorption of an electron beam. J. Fluid Mech., 74(3):497-513, 1976. 
This document was prepared as an account of work sponsored by the United States Government. While this document is believed to contain correct information, neither the United States Government nor any agency thereof, nor The Regents of the University of California, nor any of their employees, makes any warranty, express or implied, or assumes any legal responsibility for the accuracy, completeness, or usefulness of any information, apparatus, product, or process disclosed, or represents that its use would not infringe privately owned rights. Reference herein to any specific commercial product, process, or service by its trade name, trademark, manufacturer, or otherwise, does not necessarily constitute or imply its endorsement, recommendation, or favoring by the United States Government or any agency thereof, or The Regents of the University of California. The views and opinions of authors expressed herein do not necessarily state or reflect those of the United States Government or any agency thereof, or The Regents of the University of California.

Ernest Orlando Lawrence Berkeley National Laboratory is an equal opportunity employer. 